\title{
The Effects of Aerobic Training and Omega-3 Intake on $A \beta 42$, Neprilysin, and $\gamma$ - Secretase in the Hippocampus of Male Rats Alzheimer's model
}

Ali Yaghoubi ( $\nabla$ yaghoubiali65@gmail.com )

Islamic Azad University

Research

Keywords: Aerobic Training, Omega-3 Intake, amyloid- $\beta 42$, Neprilysin, $\gamma$-Secretase, Alzheimer's disease

Posted Date: May 4th, 2021

DOI: https://doi.org/10.21203/rs.3.rs-427829/v1

License: (c) (i) This work is licensed under a Creative Commons Attribution 4.0 International License.

Read Full License 


\section{Abstract}

Alzheimer's disease (AD) is characterized by excessive deposition of the amyloid- $\beta$ peptide (A $\beta$ ) in the central nervous system and reducing its level is the goal of many medications. Thus this study aimed to investigate the effect of aerobic training and omega-3 intake on $A \beta 42$, Neprilysin, and $y$-secretase in the hippocampus of male rats Alzheimer's model. 50 male wistar rats (12weeks-old and weight $222.31 \pm$ $11.91 \mathrm{~g}$ ), divided into 5 groups including, control Alzheimer's (AC), Alzheimer's with the omega-3 intake (AO), Alzheimer training (AT), Alzheimer's with omega-3 intake, and Alzheimer training (AOT) and Healthy Control $(\mathrm{HC})$. Alzheimer's disease induced by injection of homocysteine $(60 \mathrm{mM})$ into the rat brain ventricle. Training with a speed of $20 \mathrm{~m} / \mathrm{min}$ ( 60 minutes and 5 days/week on the treadmill) was applied. The supplement group was received an Omega-3 supplement $800 \mathrm{mg} / \mathrm{kg}$ of body weight, daily for eight weeks. Level of $A \beta 42, y$-secretase, and Neprilysin protein measured using ELISA method. In data analysis, a one-way ANOVA and Tukey test as a post hoc were used $(P<0.05)$. The obtained results showed that the level of $A \beta 42$ in the hippocampus of $A C$ was significantly higher than the $H C$ group $(P=0.001)$. Also, the level of the $A \beta 42$ in the hippocampus of $A C$ was significantly higher than $A O, A T$, and $A O T$ groups $(P$ values $0.001,0.007$, and 0.003 respectively). Also the $y$-Secretase level in the hippocampus of $A C$ was significantly higher than the $\mathrm{HC}$ group $(P=0.001)$. Also, the $y$-secretase levels in the hippocampus of the AC group were significantly higher than AO, AT, and AOT groups ( $P$ values, $0.002,0.001$, and 0.001 respectively). There was no significant difference between Neprilysin levels of the hippocampus of research groups $(P=0.534)$. It appears that exercise training and omega-3 consumption, can prevent amyloidogenic pathways by reducing the level of $y$-secretase, and lead to reducing the level of $A \beta$ hippocampus of Alzheimer's disease subjects. In total, aerobic exercise training and omega-3 intake can be studied as complementary therapy in Alzheimer's patients.

\section{Introduction}

Alzheimer's Disease (AD) is the most common form of dementia currently affecting over 50 million people worldwide, and over 5 million Americans (Alzheimer's Association). The prevalence of AD has increased greatly, particularly in countries that have seen an increase in life expectancy. Prevalence is below $1 \%$ in the population under 60 years of age, increasing to $40 \%$ among those older than 85 [1].

An abnormal elevation of homocysteine (Hcy) levels has been implicated as a marker for AD. Hyperhomocysteinemia is associated with increased cognitive decline in healthy older adults with a higher risk of cognitive impairment [2, 3]. In this regard, Farina et al. (2017) showed that cognitive status significantly declined over the follow-up period of research (13 months) and that was paralleled by a significant increase in homocysteine concentrations [4]. Mechanisms of increased levels of Hcy and creation of its nervous toxicity effects are not fully understood, but a possible increase in Amyloid- $\beta$ (A $\beta$ ) in the effect of increasing Hcy suggested $[5,6]$.

The complex pathology of the disease is characterized by several hallmarks, such as prominent extracellular amyloid plaques [7]. According to the amyloid cascade hypothesis, an alteration of $A \beta$ 
metabolism is the central pillar of AD pathology and crucially influences and initiates other hallmarks [8]. In $A D$, initial pathologic processes progress decades before the first cognitive symptoms appear in patients, a stage entitled preclinical Alzheimer's [9].

$A \beta$ as a monomer is a very hydrophobic peptide that is found naturally in small amounts in the brain and has 37-49 amino acids which are created by $A \beta$ precursor protein (A $\beta P P)$ proteolysis $[8,10,11]$. This peptide has no fixed physiological function and as a result of a metabolic process, can produce both amyloidogenic and non-amyloidogenic products [12]. If the AßPP, is cleaved by the enzyme $a$-Secretase, Neuroprotective piece SA $\beta P P a$ (a piece of APP by a-secretase isolated) produced and prevent the formation of $A \beta$ plaques [13]. Conversely, if fragmentation is needed for successive APP, first by $\beta$ - and then by $Y$-secretase, followed by the formation of $A \beta 42$ and causes an increase in the level of $A \beta 42$ in the nervous system $[14,15]$. Isolated parts in the $\beta$-secretase activity of $A \beta P P$, causes the production of SA $\beta P P \beta$ and finally $Y$-secretase activity on APP, followed by the production of $A \beta$. A $\beta$ production in $Y^{-}$ secretase activity, is very susceptible to oligomerize and has extremely high toxicity [16], and the deposition of protein plaques in the brain is known as one of the main causes of early and important events in the pathogenesis of $A D$ that the first to be formed in the hippocampus and is involved in the analysis of neurons in $A D$ [10]. A study on the transgenic rat (Tg) Alzheimer's, has strengthened this hypothesis that memory loss is associated with the level of $A \beta[10,17]$. Researchers have shown that $A \beta$ injected into the hippocampus of the brain, causing impaired learning and memory in rats and causes neurodegeneration and neuronal dysfunction [18] and cleaning different areas of the brain from the presence of $A \beta$, can play an important role in improving the symptoms of $A D$ [19].

According to the studies, some of the A $\beta$-degrading proteases help to adjust its level in the brain. These enzymes are mainly serine or metalloproteinase which include an Insulin-Degrading Enzyme (IDE), Neprilysin (NEP), Endothelin Converting Enzyme (ECE), Angiotensin-Converting Enzyme (ACE), and Matrix Metalloproteinase-9 (MMP-9). Among these factors, Neprilysin is the main degrading enzymes of $A \beta$ [20].

The main modifiable risk and protective factors for $A D$ are socioeconomic factors such as level of schooling; lifestyle factors such as alcohol and tobacco consumption and level of physical activity; and dietary factors such as the consumption of caffeine, antioxidants, and fatty acids $[1,21]$.

Omega-3 Fatty Acids are essential fatty acids that are the components of neuronal cell membranes in the brain [22-24]. Several studies have highlighted the neuroprotective roles of Omega-3 in neurodegenerative disorders such as Huntington's disease (HD) [25] and Parkinson's disease (PD) [21] as well as $A D$ and Mild Cognitive Impairment $(\mathrm{MCl})[26,27]$. Also, it is asserted that physical activity plays a pivotal role in the prevention of neurodegenerative disorders. Although age is a dominant risk factor for $A D$, epidemiological studies have shown that exercise may significantly decrease age-related risks for $A D$ [28-31].

Because of the shared neurobiological and physiological effects of physical activity (PA) and omega-3 intake, several human and animal studies have speculated about the additive or multiplicative benefits that might arise from combining omega-3 intake with PA [32, 33]. For example, PA may provide an avenue 
by which the effects of Docosahexaenoic acid (DHA) on cellular integrity and cognitive function are enhanced [32,34]. The combination of PA and DHA intake have additive effects on synaptic plasticity and membrane structure biomarkers in the dentate gyrus of the hippocampus, such that mice receiving both DHA and PA have greater levels of synaptic proteins than their counterparts not receiving PA [35]. However, these effects were not mirrored behaviorally. Instead, physical inactivity without DHA supplementation resulted in impaired learning compared to mice with DHA intake, PA, or both [35]. Studies in humans have not yet examined whether DHA levels moderate the effect of PA on cognitive performance in a similar way to that demonstrated in rodents.

Overall, because the effect of omega-3 intake along with aerobic training on $A \beta$ metabolism has not been well studied. Thus, this study aimed to investigate the effect of aerobic training and omega-3 intake on the level of $A \beta 42$, Neprilysin, and $y$-secretase Alzheimer's hippocampus of male rats Alzheimer's model.

\section{Material And Methods}

The current study is experimental which was conducted in the laboratory method. In this study, 50 head of adult male Wistar rats with a weight range of 100 to $150 \mathrm{~g}$ and the age of 8 weeks were used which was prepared by the Pasteur Institute in northern Iran. The rats were kept in an environment with a temperature of $22 \pm 2^{\circ} \mathrm{C}$, humidity $50 \pm 5 \%$, and the light-dark cycle of $12: 12$ hours in polycarbonate cages (5 rats per cage). After 4 weeks, 40 rats were selected for Intracerebroventricular (ICV) Injection of Hcy and 10 rats for the healthy group.

\section{Intracerebroventricular (ICV) Injection of Hcy}

After the rats were in the range of 200 to $250 \mathrm{~g}$ in weight; rats by intraperitoneal injection of ketamine and xylazine (with doses of 50 and $4 \mathrm{mg} \mathrm{kg}$ ) were anesthetized. The head of rats was placed into stereotaxic surgery and according to Paxinos and Watson atlas, within the context of their brains, the cannula was inserted and connected to the skull with dental cement. A week later connecting a cannula, Hcy solution to volume 1 microliter, was injected into rat brain ventricles by Hamilton syringe. An effective amount of Hcy for neural degeneration and Alzheimer's $0.6 \mathrm{M}$ (0.86 micrograms per mouse) is designated [36].

The shuttle box was used to assess behavioral change and ensure the induction of Alzheimer's disease. Alzheimer's rat was divided randomly into 5 groups including control Alzheimer's (AC), Alzheimer's with the omega-3 intake (AO), Alzheimer training (AT), and Alzheimer's with omega-3 intake and Alzheimer training (AOT), there was also a group called Healthy Control (HC). Because 6 head of rats died after Cannula or during the study period; the number of samples at the end of the study was 44 head.

\section{Aerobic training protocol}

Aerobic training included the entire period of 8 weeks and 5 days a week and was conducted in 3 stages. In the trading phase (first week) the rats walk every day for 10-15 minutes on a treadmill at a speed of 10 $\mathrm{m} / \mathrm{min}$. At the overload (the second and third weeks), gradually during the second week, added to the 
intensity and duration of the training, has reached the final 60 minutes with a speed of $20 \mathrm{~m} / \mathrm{min}$. In the process of preservation or stabilization (fourth to the eighth week), training continued with the same intensity to 8 weeks ended. This is equivalent intensity by 50 to 55 percent of maximum oxygen consumption in a rat $[37,38]$.

\section{Omega-3 intake}

Omega-3 receiving groups, intake $800 \mathrm{mg} / \mathrm{kg}$ of the omega-3 supplement daily by gavage for eight weeks [39]. The omega-3 supplement used included $136 \mathrm{mg} / \mathrm{ml}$ DHA and $139 \mathrm{mg} / \mathrm{ml}$ EPA [40].

All subjects, 72 hours after the last training session, with a combination of intraperitoneal injection of Ketamine (50 mg per $\mathrm{kg}$ ) and Xylazine (4 $\mathrm{mg}$ per $\mathrm{kg}$ ) were anesthetized [36]. To collect samples of the hippocampus, the subjects were isolated in the neck by cutting pliers, using the knife, split the skull and brain were removed with caution. Healthy brain by the surgery was split exactly in half and given the coordinates of the hippocampus using Paxinos atlas, the hippocampus was removed from the limbic system. Hippocampus samples collected for subsequent measurements were stored at $-80^{\circ} \mathrm{C}$. It should be noted, all procedures consisted of a cannula, inducing $A D$ inducing, the training protocol and killing and biopsy were done according to the regulations of the biological research ethics committee of Mazandaran University.

To measure hippocampal levels of $A \beta 42$, $Y$-secretase, and Neprilysin, initially $50 \mathrm{mg}$ hippocampus tissues were placed in cold saline citrate buffer solution. Then the tissue was homogeneous by microhomogenizer for 10 minutes. By homogeneous tissue was centrifuged and the supernatant was transferred into eppendorf. This product was used to measure the level of $A \beta 42, \gamma$-secretase, and Neprilysin in hippocampal tissue. Hippocampus $A \beta 42$ levels were measured by ELISA using research kits for rats (manufactured by Cusabio Biotech Wuhan, China) and following the manufacturer's instructions. The sensitivity measurement kit was $0.225 \mathrm{pg} / \mathrm{mL}$ and the coefficient of variation was 8.20 percent. Neprilysin hippocampus levels were measured by ELISA using research kits for rats (manufactured by Wuhan Cusabio Biotech) according to the manufacturer's instructions. The sensitivity measurement kit was $11.75 \mathrm{pg} / \mathrm{mL}$ and the intra-coefficient was 8.70 percent. The level of $\gamma$ - secretase hippocampus were measured by ELISA using research kits for rats (manufactured by Sunlong, China) and following the manufacturer's instructions. The sensitivity measurement kit was $0.60 \mathrm{pg} / \mathrm{mL}$ and the coefficient of variation within the test was 7.40 percent.

Shapiro-Wilk test was used to assess the normality of the data and the Leven test was used to check equal variances. After the assumption of the normal distribution and equality of variances, ANOVA and Tukey test were used for statistical analysis and data comparison between groups, and the Pearson correlation coefficient was used to examine relationships between variables. All statistical calculations were performed using SPSS23 statistical software at a significant level of P囚0.05.

\section{Results}


Table 1 shows the mean and standard deviation of rats' weight before and after 8 weeks of aerobic research groups.

Table 1

Mean and standard deviation of rat's weight before and after 8 weeks of aerobic training in the research groups *

\begin{tabular}{|llll|}
\hline Research groups & $\begin{array}{l}\text { Initial weight } \\
(\mathbf{g})\end{array}$ & $\begin{array}{l}\text { Final weight } \\
(\mathbf{g})\end{array}$ & $\begin{array}{l}\text { Arrival to dark areas } \\
(\mathbf{S})\end{array}$ \\
\hline HC & $217.13 \pm 22.90$ & $325.38 \pm 11.90$ & $183.82 \pm 78.29$ \\
\hline AC & $220.9 \pm 63.90$ & $301.24 \pm 25.90$ & $25.21 \pm 50.78$ \\
\hline AO & $227.11 \pm 25.97$ & $303.29 \pm 13.51$ & $23.18 \pm 75.20$ \\
\hline AT & $221.11 \pm 33.70$ & $327.44 \pm 33.90$ & $23.15 \pm 44.96$ \\
\hline AOT & $227.11 \pm 63.90$ & $307.31 \pm 38.70$ & $25.25 \pm 0.52$ \\
\hline * Numbers are expressed as the mean \pm standard deviation. \\
\hline
\end{tabular}

After verification of normality and using one-way analysis of variance showed that there was no significant difference between the weight of the rats in the first $(P=0.456, F=0.968)$ and end $(P=0.446$, $F=0.983$ ) training courses between groups.

After verification of normality and using one-way analysis of variance showed that there is a significant difference between the latency to enter the dark area between groups $(P=0.001, F=19.21)$. The findings from the post-hoc test comparing pair's latency to enter the dark compartment in different groups showed that the index at baseline in the group of healthy control was significantly upper than Alzheimer's group $(P=0.001)$. There was no significant difference between the latency to enter the dark area between groups between Alzheimer's group $(P>0.05)$.

Table 2 showed the test results to compare the effects of aerobic training and omega- 3 intake on the levels of $A \beta 42, Y$ - secretase, Neprilysin in the research groups. The results in Table 2 , there was a significant difference between the average levels of $A \beta 42$ and $\gamma$-secretase in study groups ( $P$ values 0.001 and 0.001 respectively), but there was no significant difference between the mean of Neprilysin in the research groups $(P=0.534)$. 
Table 2

Comparison of index levels of the study (mean \pm SD) in the hippocampus of study groups and the results of the analysis of variance

\begin{tabular}{|c|c|c|c|}
\hline Group & $\begin{array}{l}A \beta_{42} \\
) p g / m g \text { tissue( }\end{array}$ & $\begin{array}{l}\text { Y- secretase } \\
\text { )pg/mg tissue( }\end{array}$ & $\begin{array}{l}\text { Neprilysin } \\
\text { )ng/mg tissue }\end{array}$ \\
\hline $\mathrm{HC}$ & $48.33 \pm 16.98$ & $46.50 \pm 8.15$ & $7.19 \pm 1.72$ \\
\hline$A C$ & $102.01 \pm 20.73$ & $87.03 \pm 18.62$ & $7.48 \pm 1.31$ \\
\hline $\mathrm{AO}$ & $59.87 \pm 18.91$ & $58.97 \pm 13.35$ & $9.45 \pm 3.07$ \\
\hline AT & $70.22 \pm 24.50$ & $37.02 \pm 8.29$ & $8.72 \pm 2.44$ \\
\hline AOT & $66.25 \pm 15.13$ & $42.96 \pm 15.47$ & $9.65 \pm 3.66$ \\
\hline $\mathrm{F}$ & 8.466 & 21.829 & 0.744 \\
\hline $\mathrm{P}$ & $0.001^{\&}$ & $0.001^{\&}$ & 0.534 \\
\hline
\end{tabular}

\section{Aß42 level of the hippocampus}

Information available in Fig. 1 shows the level of $A \beta 42$ in the hippocampus of $A C$ was significantly higher than the $H C$ group $(P=0.001)$. Also, the level of the $A \beta 42$ in the hippocampus of $A C$ was significantly higher than $A O, A T$, and $A O T$ groups ( $P$ values $0.001,0.007$, and 0.003 respectively). On the other hand, there was no significant difference between $A O, A T$, and AOT groups $(P>0.05)$. Figure 1 shows the results of research comparing the levels of $A \beta 42$ hippocampus groups after 8 weeks of Aerobic training and omega-3 intake.

$Y$-Secretase level of the hippocampus: The results showed that the $Y$-Secretase level in the hippocampus of $A C$ was significantly higher than the $\mathrm{HC}$ group $(P=0.001)$. Also, the $\gamma$ - secretase levels in the hippocampus of the AC group were significantly higher than AO, AT, and AOT groups ( $P$ values, 0.002 , 0.001 , and 0.001 respectively). $Y$ - secretase levels in the hippocampus of the $A O$ group were significantly higher than AT group $(P=0.019)$. But there was no significant difference between hippocampus $Y^{-}$ secretase levels in AT and AOT group $(P=0.848)$. Figure 2 shows the results of research comparing the levels of $\gamma$ - secretase hippocampus of research groups after 8 weeks of aerobic training and omega-3 intake.

\section{Neprilysin level of the hippocampus}

Based on the data presented in Table 2, there was no significant difference between the Neprilysin level of the hippocampus of research groups $(P=0.534)$. 


\section{Discussion}

We found ICV Injection of Hcy is associated with high A 42 and $\gamma$-Secretase levels of the hippocampus. Also, aerobic training and Omega-3 intake lowered $A \beta 42$ and $\gamma$-Secretase levels of the hippocampus in AD subjects.

It is well established that elevated plasma homocysteine and disturbed homocysteine metabolism are risk factors for $A D$ [1]. However, the exact pathophysiological mechanisms linking high homocysteine levels with $A D$ are not yet clear. Several potential mechanisms resulting in harmful effects of this amino acid in the brain have been proposed, including oxidative stress [41], cerebrovascular damage [42], DNA damage [43], and activation of N-methyl-D-aspartate receptors [44]. Several showed that disturbed homocysteine metabolism is related to increased CSF levels of sAPP forms and AB42, and may contribute to the accumulation of amyloid pathology in the brain through increasing $y$-secretase pathway $[43,45]$. For example, Lin et al (2009) show that Hcy increases the production of $A \beta$ possibly by increased expression of APP as well as induction of hypomethylation of APP and PS1 gene promoters [46], whereas in rats hyperhomocysteinemia increases cerebral $A \beta$ production by phosphorylation of amyloid precursor protein and enhancing expression of $\gamma$-secretase [47]. More recently, mice with diet-induced hyperhomocysteinemia were shown to have elevated brain $A \beta$ levels and amyloid deposition and it was suggested that this association is mediated by the activation of the $y$-secretase pathway [48]. These previous reports provide a possible explanation of the biochemical process, connecting disturbed homocysteine metabolism and increased CSF levels of sAPP forms and A 342.

Based on the evidence, both microglia and astrocytes secreted $A \beta$ protein [49]. Senile plaques, mainly composed of peptides $A \beta$ is that its main role has widely proven in the pathogenesis of Alzheimer's disease $[49,50]$. In particular, increased levels of $A \beta$ deposition in plaques outside the cell and cause synaptic dysfunction, neuronal network dysfunction, mitochondrial dysfunction, neuronal cell death, and memory loss $[49,51,52]$. Although the mechanism of neurotoxicity caused by $A \beta$ is not yet clear, but widely proven that the accumulation of $A \beta$ peptide in the brain causes induction of oxidative stress and neuroinflammation $[49,50]$. In vitro studies have shown that injection of $A \beta 42$ in primary hippocampal neurons leads to increased planting in the indices of oxidative stress and neurotoxicity $[53,54]$. This peptide associated with oxidative stress caused by $A \beta$, adding vitamin $E$ as an antioxidant, significantly dampens the effects of oxidative stress and neurotoxicity induced $A \beta 42$ [53], which suggests that the ability of the peptide $A \beta 42$-induced neurotoxicity toxic oxidative stress-mediated. This regard indicated that $A \beta 40$ injection into the brain of rats is associated with the induction of free radical damage and changes in antioxidant defense such as glutathione depletion in the prefrontal cortex and hippocampus of rats [55].

It has been shown that $A \beta$ acts as an inflammatory agent and causes inflammatory mediators such as cytokines and complement components activated brain areas to increase the risk of Alzheimer's. Besides, the presence of activated microglia and astroglia around senile plaques supports the role of $A \beta$ in inflammation. Microglia and astrocytes may have a neuroprotective role by swallowing and clearing $A \beta$ 
aggregates in the brain $[56,57]$. Although it can mediate neurotoxicity effects through the release of proinflammatory cytokines, chemokines, ROS, and protein supplements [56]. Besides, APP expression by IL-1 (as an inflammatory cytokine) is increased and thus enhancing amyloidosis and leads to a vicious cycle [58]. Now, there is growing evidence that low to moderate-intensity training is an important factor for neural degenerative diseases [59].

In the present study AB42 levels in the hippocampus of AT group were significantly lower than the AC group and no difference was observed between the levels of the $A \beta 42$ hippocampus of $A T, A O, A O T$, and $\mathrm{HC}$ groups.

The amyloid cascade hypothesis and the law of mass action, each level balance between the synthesis and degradation of proteins in the body/cleans it and $A \beta$ levels in the brain balance between the synthesis and degradation/clean it [16].

In the present study to study changes in $A \beta$ production in the training groups, the $\gamma$ - secretase was evaluated and it was found that the level of $\gamma$ - secretase of AC group was significantly higher than $\mathrm{HC}$ group. Thus increasing A 342 levels in the hippocampus of Alzheimer's rat compared to a healthy rat can be caused by increased levels of $\gamma$ - secretase in hippocampal them.

The results of this study showed that the level of $y$ - secretase in the hippocampus of AT and AOT was significantly lower than the AC group. The results showed that there was no significant difference between Neprilysin hippocampus levels of research groups. As mentioned before, Neprilysin is considered as the main degrading enzyme $A \beta$ and as a regulator has raised concentrations of $A \beta$ in the brain functional surfaces $[60,61]$. Failure to raise the level of Neprilysin means no change in the demolition/clean A 442 from the hippocampus of Alzheimer's subjects which seeks to increase the level of the index and can lead to the development of Alzheimer's disease risk. Kang and Cho (2014) examined the effect of 6 weeks of treadmill training on insulin signaling and brain $A \beta$ levels in the Alzheimer induce rat by streptozotocin. Their results showed a significant decrease in A $\beta 42$ levels and increase insulin signaling in the brains of rat of Alzheimer training compared to Alzheimer control groups. They suggested that reducing insulin signaling is associated with elevated levels of $\gamma$-secretase, which leads to an increase in $A \beta$ and improves insulin signaling caused by six weeks of training on a treadmill, might be a moderation of $\gamma$ - secretase to reduce $A \beta$ [62]. Also, Liu et al (2013) showed that the number and size of $A \beta$ plaques in the hippocampus of a rat with Alzheimer's disease five months after the treadmill training was significantly reduced. The levels of A 342 , tau protein, and PS1 expression decreased significantly as a result of training on the treadmill. Besides, a reduction in the levels of CTFs and SABPP $\beta$ in training transgenic rats was observed. The researchers concluded that perhaps treadmill workouts prevent the amyloidogenic pathway and the breakdown of APP to be adjusted to non-amyloidogenic[63]. Also, Kang et al (2013) stated that 12 weeks of treadmill training to prevent the disorder gene mutation PS2 and reduced the accumulation of $A \beta$ by inhibiting the activity of $\beta$ - secretase and its products [64]. Besides, Um et al (2008) also showed that 16 weeks of treadmill training causes a significant decrease in $A \beta 42$ in the brain of rats with Alzheimer's protein levels [65]. The results also showed that training with two 
different intensities by reducing the $\gamma$ - secretase that is amyloidogenic pathway causes a decrease in $A \beta$ that is consistent with the results obtained from the research.

There are several theories about changes in $A \beta$ levels and metabolism (synthesis and degradation) as a result of training. According to the physical activity mediates many gene products both at the mRNA and protein level, Inducer of anatomical changes, chemical and electrophysiological nerve, is enhancer the plasticity of neurons [66], multiple paths are likely enabled to adjust the level of $A \beta$ directly or indirectly. Adlard et al (2005) stated that exercise training can probably mediate the metabolism of APP and A $\beta$ cascade in the brain to reduce the production of $A \beta$ (decreasing amyloidogenic activity) which is independent of the neprilysin and IDE [67]. The second possibility is that exercise directly modulates the APP metabolism by increasing the activity of neurons. For example, processing of APP can be completed by mitogen-activated protein kinase (MAPK) and phospholipase $C$ and has proved that these pathways are activated through exercise [68]. On the other hand, cholinergic activity increases with exercise and cholinergic systems by physical activity involved in neuronal plasticity induced by exercise [66].

Mentioned is probably exercise can improve behavioral disorders by reducing the number of peptide A 342 by increasing the production of neurotrophic factors (NGF, BDNF, and IGF-1) which are important for neuronal survival, a proliferation of neuronal and synaptic plasticity [69]. Also, physical activity reduces $A \beta$ plaque and improves spatial learning (three-dimensional), memory, synaptic plasticity, and nerve tissue of Alzheimer's disease rat [70]. Several studies have reported that the cytoplasmic surface of apoptotic markers such as Cytochrome $\mathrm{C}$, caspases-9, caspases-3, and Bax protein was significantly decreased in the brains of active Alzheimer's rat to the inactive Alzheimer's rat. In Alzheimer's rats, decreased pro-apoptotic proteins, including cytochrome $\mathrm{C}$ and boxes with physical activity, possibly by preventing apoptotic pathways related to caspases, are systematically associated with lower levels of protein $A \beta[65,71]$. However, Park et al $(2010)$ has provided a cyclic process stimulate $A \beta$ in inflammation and suggested that signaling TNF-a, ultimately leads to the production of $A \beta$ peptides causing produce new pathogenic $A \beta$ peptides and increase its production and thus leads to Alzheimer's disease that can create a stronger cycle [72]. In this regard, Nichol et al (2008) demonstrated that inflammatory markers (IL-1 $\beta$ and TNF- $\alpha$ ) in the hippocampus of Alzheimer's transgenic rat was higher than the healthy rat and the level of anti-inflammatory agents (IFN- $y$ and MIP-1a) in Alzheimer's transgenic rat was lower than the healthy rat. After 3 weeks of training, the levels of IL-1 $\beta$ and TNF- $\alpha$ decreased and was close to the normal group that this reduction was associated with an increase in IFN- $\gamma$ and MIP-1a. Also a significant decrease in the levels of $A \beta 40$ solution and fibrillar $A \beta$ solution in the cortex of Alzheimer's transgenic rat by 3 weeks optional practice was observed [73]. Also, Um et al (2008) showed that the SOD-1 protein and catalase in the brains of active Alzheimer's rat has a significant increase compared to the inactive. Exercise causes increased levels of these defense indexes that these changes are associated with reduced apoptotic protein (Cytochrome C, caspases-9, caspases-3, and bax) and an increase in Hsp70 and BDNF which is induced by regular physical activity in the brain and then was mediated by peptides Aß42 clinical reduced in rat with Alzheimer's disease [65]. It has been shown that 16 weeks of treadmill exercise combined with a-lipoic acid, decreased levels of brain A $\beta 42$ transgenic Alzheimer's rat. The researchers reported that increased oxidative stress, is one of the main factors involved in Alzheimer's 
which leads to increased production of ROS and causes the destruction of cellular structures, and ultimately apoptosis increased the production of $A \beta$. Exercise alone and in combination with a-lipoic acid supplementation resulted in increased levels of oxidative stress and antioxidants as immunosuppressive agents and finally resulting in reduced apoptotic index and $A \beta$ [71]. Possible mechanisms in this regard include the reduction of oxidative stress and increase antioxidant defense enzyme activity act as an agent enhancing the $a$-secretase and inhibitor $\beta$ - and $\gamma$ - secretase and so the processing of APP to conduct the Non-amyloidogenic pathway [74]. The improvement in $A \beta$ and $\gamma$-secretase levels in the present study may also be due to the reduction of oxidative stress and the improvement of antioxidant defense through omega-3 supplementation and aerobic exercise.

\section{Conclusion}

However, many researchers have proposed using different drugs for the treatment of Alzheimer's disease, but generally, each has serious side effects. Thus, lifestyle changes such as exercise and nutrition can be used as a method to complement the medical treatment and also lead to a reduction in the side effects of high doses of medication. Since many drug treatment efforts for Alzheimer, focused to inhibit $\mathrm{Y}^{-}$ secretase and reduction of $A \beta$ and according to the survey results, it appears that exercise training and omega- 3 consumption, can prevent amyloidogenic pathways by reducing the level of $\gamma$ - secretase, and lead to reducing the level of $A \beta$ hippocampus of Alzheimer's disease subjects. In total, aerobic exercise training and omega-3 intake can be studied as complementary therapy in Alzheimer's patients.

\section{Declarations}

Ethics approval and consent to participate: all procedures consisted of a cannula, inducing AD inducing, the training protocol and killing and biopsy were done according to the regulations of the biological research ethics committee of Mazandaran University, and my manuscript does not contain any individual person's data thus not applicable.

Availability of Data and Materials: Please contact author for data requests.

Funding: I has NO affiliation with or involvement in any organization or entity with any financial interest

Competing interests: The authors declare that they have no competing interests.

Authors' contributions: only 1 person do all step of this paper.

Acknowledgments: I wish to thank all my collaborators of the biology department and exercise physiology laboratory of Mazandaran University and also special thanks to my dear friend Faraz Motaghedi for his valuable assistance.

\section{References}


1. Beydoun MA, Beydoun HA, Gamaldo AA, Teel A, Zonderman AB, Wang Y. Epidemiologic studies of modifiable factors associated with cognition and dementia: systematic review and meta-analysis. BMC Public Health. 2014;14(1):643.

2. Morris MS. The role of $B$ vitamins in preventing and treating cognitive impairment and decline. Advances in nutrition. 2012;3(6):801-12.

3. Smith AD, Smith SM, De Jager CA, Whitbread P, Johnston C, Agacinski G, et al. Homocysteinelowering by $B$ vitamins slows the rate of accelerated brain atrophy in mild cognitive impairment: a randomized controlled trial. PloS one. 2010;5(9):e12244.

4. Farina N, Jernerén F, Turner $\mathrm{C}$, Hart $\mathrm{K}$, Tabet N. Homocysteine concentrations in the cognitive progression of Alzheimer's disease. Exp Gerontol. 2017;99:146-50.

5. Pacheco-Quinto J, de Turco EBR, DeRosa S, Howard A, Cruz-Sanchez F, Sambamurti K, et al. Hyperhomocysteinemic Alzheimer's mouse model of amyloidosis shows increased brain amyloid $\beta$ peptide levels. Neurobiol Dis. 2006;22(3):651-6.

6. Cascalheira JF, João SS, Pinhanços SS, Castro R, Palmeira M, Almeida S, et al. Serum homocysteine: interplay with other circulating and genetic factors in association to Alzheimer's type dementia. Clinical biochemistry. 2009;42(9):783-90.

7. Sharma P, Srivastava P, Seth A, Tripathi PN, Banerjee AG, Shrivastava SK. Comprehensive review of mechanisms of pathogenesis involved in Alzheimer's disease and potential therapeutic strategies. Progress in neurobiology. 2019;174:53-89.

8. Selkoe DJ, Hardy J. The amyloid hypothesis of Alzheimer's disease at 25 years. EMBO molecular medicine. 2016;8(6):595-608.

9. Bateman RJ, Xiong C, Benzinger TL, Fagan AM, Goate A, Fox NC, et al. Clinical and biomarker changes in dominantly inherited Alzheimer's disease. N Engl J Med. 2012;367:795-804.

10. Selkoe DJ. Alzheimer's disease: genotypes, phenotype, and treatments. Science. 1997;275(5300):630.

11. Gispen WH, Biessels G-J. Cognition and synaptic plasticity in diabetes mellitus. Trends in neurosciences. 2000;23(11):542-9.

12. Fraser ON, Bugnyar T. Ravens reconcile after aggressive conflicts with valuable partners. PLoS One. $2011 ; 6(3)$.

13. Pearson HA, Peers C. Physiological roles for amyloid $\beta$ peptides. J Physiol. 2006;575(1):5-10.

14. Seubert P, Oltersdorf T, Lee MG, Barbour R, Blomquist C, Davis DL, et al. Secretion of $\beta$-amyloid precursor protein cleaved at the amino terminus of the $\beta$-amyloid peptide. Nature. 1993;361(6409):260-3.

15. Wilson CA, Doms RW, Lee VM. Intracellular APP, Processing. and A [beta] Production in Alzheimer Disease. Journal of Neuropathology Experimental Neurology. 1999;58(8):787-94.

16. Haass C, Selkoe DJ. Soluble protein oligomers in neurodegeneration: lessons from the Alzheimer's amyloid $\beta$-peptide. Nature reviews Molecular cell biology. 2007;8(2):101-12. 
17. Podolski IY, Podlubnaya Z, Kosenko E, Mugantseva E, Makarova E, Marsagishvili L, et al. Effects of Hydrated Forms of C60 Fullerene on Amyloidî $\square$-Peptide Fibrillization In Vitro and Performance of the Cognitive Task. J Nanosci Nanotechnol. 2007;7(4-5):1479-85.

18. Yamada K, Nabeshima T. Animal models of Alzheimer's disease and evaluation of anti-dementia drugs. Pharmacol Ther. 2000;88(2):93-113.

19. Bliss TV, Collingridge GL. A synaptic model of memory: long-term potentiation in the hippocampus. Nature. 1993;361(6407):31-9.

20. Grimm MO, Mett J, Stahlmann CP, Haupenthal VJ, Zimmer VC, Hartmann T. Neprilysin and A $\beta$ clearance: impact of the APP intracellular domain in NEP regulation and implications in Alzheimer's disease. Frontiers in aging neuroscience. 2013;5.

21. Barros AS, Crispim RYG, Cavalcanti JU, Souza RB, Lemos JC, Cristino Filho G, et al. Impact of the Chronic Omega-3 Fatty Acids Supplementation in Hemiparkinsonism Model Induced by 6Hydroxydopamine in Rats. Basic Clin Pharmacol Toxicol. 2017;120(6):523-31.

22. Laye S, Nadjar A, Joffre C, Bazinet RP. Anti-inflammatory effects of omega-3 fatty acids in the brain: physiological mechanisms and relevance to pharmacology. Pharmacological reviews. 2018;70(1):12-38.

23. Echeverría F, Valenzuela R, Hernandez-Rodas MC, Valenzuela A. Docosahexaenoic acid (DHA), a fundamental fatty acid for the brain: New dietary sources. Prostaglandins Leukot Essent Fatty Acids. 2017;124:1-10.

24. Bazinet RP, Layé S. Polyunsaturated fatty acids and their metabolites in brain function and disease. Nat Rev Neurosci. 2014;15(12):771-85.

25. Clifford JJ, Drago J, Natoli AL, Wong JY, Kinsella A, Waddington JL, et al. Essential fatty acids given from conception prevent topographies of motor deficit in a transgenic model of Huntington's disease. Neuroscience. 2002;109(1):81-8.

26. Grimm MO, Michaelson DM, Hartmann T. Omega-3 fatty acids, lipids, and apoE lipidation in Alzheimer's disease: a rationale for multi-nutrient dementia prevention. J Lipid Res. 2017;58(11):2083-101.

27. Olivera-Perez HM, Lam L, Dang J, Jiang W, Rodriguez F, Rigali E, et al. Omega-3 fatty acids increase the unfolded protein response and improve amyloid- $\beta$ phagocytosis by macrophages of patients with mild cognitive impairment. FASEB J. 2017;31(10):4359-69.

28. Lindsay J, Laurin D, Verreault R, Hébert R, Helliwell B, Hill GB, et al. Risk factors for Alzheimer's disease: a prospective analysis from the Canadian Study of Health and Aging. Am J Epidemiol. 2002;156(5):445-53.

29. Richards M, Hardy R, Wadsworth ME. Does active leisure protect cognition? Evidence from a national birth cohort. Soc Sci Med. 2003;56(4):785-92.

30. Larson EB, Wang L, Bowen JD, McCormick WC, Teri L, Crane P, et al. Exercise is associated with reduced risk for incident dementia among persons 65 years of age and older. Ann Intern Med. 2006;144(2):73-81. 
31. Geda YE, Roberts RO, Knopman DS, Christianson TJ, Pankratz VS, Ivnik RJ, et al. Physical exercise, aging, and mild cognitive impairment: a population-based study. Arch Neurol. 2010;67(1):80-6.

32. Gómez-Pinilla F, Feng C. Molecular mechanisms for the ability of exercise supporting cognitive abilities and counteracting neurological disorders. Functional neuroimaging in exercise and sport sciences. Springer; 2012. pp. 25-43.

33. Leckie RL, Manuck SB, Bhattacharjee N, Muldoon MF, Flory JM, Erickson KI. Omega-3 fatty acids moderate effects of physical activity on cognitive function. Neuropsychologia. 2014;59:103-11.

34. Köbe T, Witte AV, Schnelle A, Lesemann A, Fabian S, Tesky VA, et al. Combined omega-3 fatty acids, aerobic exercise and cognitive stimulation prevents decline in gray matter volume of the frontal, parietal and cingulate cortex in patients with mild cognitive impairment. Neuroimage. 2016;131:22638.

35. Chytrova G, Ying Z, Gomez-Pinilla F. Exercise contributes to the effects of DHA dietary supplementation by acting on membrane-related synaptic systems. Brain research. 2010;1341:3240.

36. Hosseinzadeh S, Roshan VD, Pourasghar M. Effects of intermittent aerobic training on passive avoidance test (shuttle box) and stress markers in the dorsal hippocampus of wistar rats exposed to administration of homocysteine. Iranian journal of psychiatry behavioral sciences. 2013;7(1):37.

37. Garekani ET, Mohebbi H, Kraemer RR, Fathi R. Exercise training intensity/volume affects plasma and tissue adiponectin concentrations in the male rat. Peptides. 2011;32(5):1008-12.

38. Fathei M. The effect of eight weeks aerobic exercise on thyroid hormones in female rats with polycystic ovary syndrome. International journal of sport studies. 2014;4.

39. Gama CS, Canever L, Panizzutti B, Gubert C, Stertz L, Massuda R, et al. Effects of omega-3 dietary supplement in prevention of positive, negative and cognitive symptoms: a study in adolescent rats with ketamine-induced model of schizophrenia. Schizophr Res. 2012;141(2-3):162-7.

40. Ma H, Wang J, Wang J, Li Y, Li J. Fish oil ameliorates the allograft arteriosclerosis of intestine on rats. Pediatric transplantation. 2007;11(2):173-9.

41. Obeid R, Herrmann W. Mechanisms of homocysteine neurotoxicity in neurodegenerative diseases with special reference to dementia. FEBS Lett. 2006;580(13):2994-3005.

42. Kamat P, Vacek J, Kalani A, Tyagi N. Homocysteine induced cerebrovascular dysfunction: a link to Alzheimer's disease etiology. The open neurology journal. 2015;9:9.

43. Pi T, Liu B, Shi J. Abnormal Homocysteine Metabolism: An Insight of Alzheimer's Disease from DNA Methylation. Behavioural Neurology. 2020;2020.

44. Tawfik A, Mohamed R, Kira D, Alhusban S, Al-Shabrawey M. N-Methyl-D-aspartate receptor activation, novel mechanism of homocysteine-induced blood-retinal barrier dysfunction. J Mol Med. 2021;99(1):119-30.

45. Oikonomidi A, Lewczuk P, Kornhuber J, Smulders Y, Linnebank M, Semmler A, et al. Homocysteine metabolism is associated with cerebrospinal fluid levels of soluble amyloid precursor protein and amyloid beta. Journal of neurochemistry. 2016;139(2):324-32. 
46. Lin H-C, Hsieh H-M, Chen Y-H, Hu M-L. S-Adenosylhomocysteine increases $\beta$-amyloid formation in BV-2 microglial cells by increased expressions of $\beta$-amyloid precursor protein and presenilin 1 and by hypomethylation of these gene promoters. Neurotoxicology. 2009;30(4):622-7.

47. Zhang C-E, Wei W, Liu Y-H, Peng J-H, Tian Q, Liu G-P, et al. Hyperhomocysteinemia increases $\beta$ amyloid by enhancing expression of $\gamma$-secretase and phosphorylation of amyloid precursor protein in rat brain. Am J Pathol. 2009;174(4):1481-91.

48. Li JG, Chu J, Barrero C, Merali S, Praticò D. Homocysteine exacerbates $\beta$-amyloid pathology, tau pathology, and cognitive deficit in a mouse model of Alzheimer disease with plaques and tangles. Ann Neurol. 2014;75(6):851-63.

49. Uslu S, Akarkarasu ZE, Ozbabalik D, Ozkan S, Çolak O, Demirkan ES, et al. Levels of amyloid beta-42, interleukin-6 and tumor necrosis factor-alpha in Alzheimer's disease and vascular dementia. Neurochem Res. 2012;37(7):1554-9.

50. Souza LC, Carlos Filho B, Goes AT, Del Fabbro L, de Gomes MG, Savegnago L, et al. Neuroprotective Effect of Physical Exercise in a Mouse Model of Alzheimer's Disease Induced by $\beta$-Amyloid1-40 Peptide. Neurotox Res. 2013;24(2):148-63.

51. Capetillo-Zarate E, Gracia L, Tampellini D, Gouras GK. Intraneuronal A $\beta$ accumulation, amyloid plaques, and synapse pathology in Alzheimer's disease. Neurodegenerative Diseases. 2012;10(14):56-9.

52. Cavallucci V, D’Amelio M, Cecconi F. A $\beta$ toxicity in Alzheimer's disease. Mol Neurobiol. 2012;45(2):366-78.

53. Boyd-Kimball D, Mohmmad Abdul H, Reed T, Sultana R, Butterfield DA. Role of phenylalanine 20 in Alzheimer's amyloid $\beta$-peptide (1-42)-induced oxidative stress and neurotoxicity. Chem Res Toxicol. 2004;17(12):1743-9.

54. Nishida Y, Ito S, Ohtsuki S, Yamamoto N, Takahashi T, Iwata N, et al. Depletion of vitamin E increases amyloid $\beta$ accumulation by decreasing its clearances from brain and blood in a mouse model of Alzheimer disease. J Biol Chem. 2009;284(48):33400-8.

55. Prediger RD, Franco JL, Pandolfo P, Medeiros R, Duarte FS, Di Giunta G, et al. Differential susceptibility following $\beta$-amyloid peptide-(1-40) administration in C57BL/ 6 and Swiss albino mice: Evidence for a dissociation between cognitive deficits and the glutathione system response. Behav Brain Res. 2007;177(2):205-13.

56. Lee CD, Landreth GE. The role of microglia in amyloid clearance from the AD brain. J Neural Transm. 2010;117(8):949-60.

57. Agostinho P, Cunha A, Oliveira R. C. Neuroinflammation, oxidative stress and the pathogenesis of Alzheimer's disease. Curr Pharm Design. 2010;16(25):2766-78.

58. Rogers JT, Lahiri DK. Metal and inflammatory targets for Alzheimer's disease. Curr Drug Targets. 2004;5(6):535-51.

59. Paillard T, Rolland Y, de Souto Barreto P. Protective effects of physical exercise in Alzheimer's disease and Parkinson's disease: a narrative review. Journal of clinical neurology. 2015;11(3):212-9. 
60. Nalivaeva N, Belyaev N, Zhuravin I, Turner A. The Alzheimer's amyloid-degrading peptidase, neprilysin: can we control it? International Journal of Alzheimer's Disease. 2012;2012.

61. Iwata N, Tsubuki S, Takaki Y, Shirotani K, Lu B, Gerard NP, et al. Metabolic regulation of brain A $\beta$ by neprilysin. Science. 2001;292(5521):1550-2.

62. Kang EB, Cho JY. Effects of treadmill exercise on brain insulin signaling and $\beta$-amyloid in intracerebroventricular streptozotocin induced-memory impairment in rats. Journal of Exercise Nutrition Biochemistry. 2014;18(1):89.

63. Liu H-I, Zhao G, Zhang H. Long-term treadmill exercise inhibits the progression of Alzheimer's disease-like neuropathology in the hippocampus of APP/PS1 transgenic mice. Behav Brain Res. 2013;256:261-72.

64. Kang E-B, Kwon I-S, Koo J-H, Kim E-J, Kim C-H, Lee J, et al. Treadmill exercise represses neuronal cell death and inflammation during $A \beta$-induced $E R$ stress by regulating unfolded protein response in aged presenilin 2 mutant mice. Apoptosis. 2013;18(11):1332-47.

65. Um HS, Kang EB, Leem YH, Cho IH, Yang CH, Chae KR, et al. Exercise training acts as a therapeutic strategy for reduction of the pathogenic phenotypes for Alzheimer's disease in an NSE/ APPswtransgenic model. Int J Mol Med. 2008;22(4):529-39.

66. Cotman CW, Berchtold NC. Exercise: a behavioral intervention to enhance brain health and plasticity. Trends in neurosciences. 2002;25(6):295-301.

67. Adlard PA, Perreau VM, Pop V, Cotman CW. Voluntary exercise decreases amyloid load in a transgenic model of Alzheimer's disease. The Journal of Neuroscience. 2005;25(17):4217-21.

68. Lu B, Chow A. Neurotrophins and hippocampal synaptic transmission and plasticity. J Neurosci Res. 1999;58(1):76-87.

69. Trejo JL, Carro E, Torres-Alemán I. Circulating insulin-like growth factor I mediates exercise-induced increases in the number of new neurons in the adult hippocampus. The Journal of neuroscience. 2001;21(5):1628-34.

70. Nichol KE, Parachikova Al, Cotman CW. Three weeks of running wheel exposure improves cognitive performance in the aged Tg2576 mouse. Behav Brain Res. 2007;184(2):124-32.

71. Um HS, Kang EB, Cho IH, Kim CH, Cho JS, Hwang DY. The combination of exercise training and alipoic acid treatment has therapeutic effects on the pathogenic phenotypes of Alzheimer's disease in NSE/APPsw-transgenic mice. Int J Mol Med. 2010;25(3):337-46.

72. Park KM, Bowers WJ. Tumor necrosis factor-alpha mediated signaling in neuronal homeostasis and dysfunction. Cellular signalling. 2010;22(7):977-83.

73. Nichol KE, Poon WW, Parachikova Al, Cribbs DH, Glabe CG, Cotman CW. Exercise alters the immune profile in Tg2576 Alzheimer mice toward a response coincident with improved cognitive performance and decreased amyloid. J Neuroinflammation. 2008;5(13):2094-5.

74. Shimmyo Y, Kihara T, Akaike A, Niidome T, Sugimoto $H$. Epigallocatechin-3-gallate and curcumin suppress amyloid beta-induced beta-site APP cleaving enzyme-1 upregulation. Neuroreport. 2008;19(13):1329-33. 
Figures

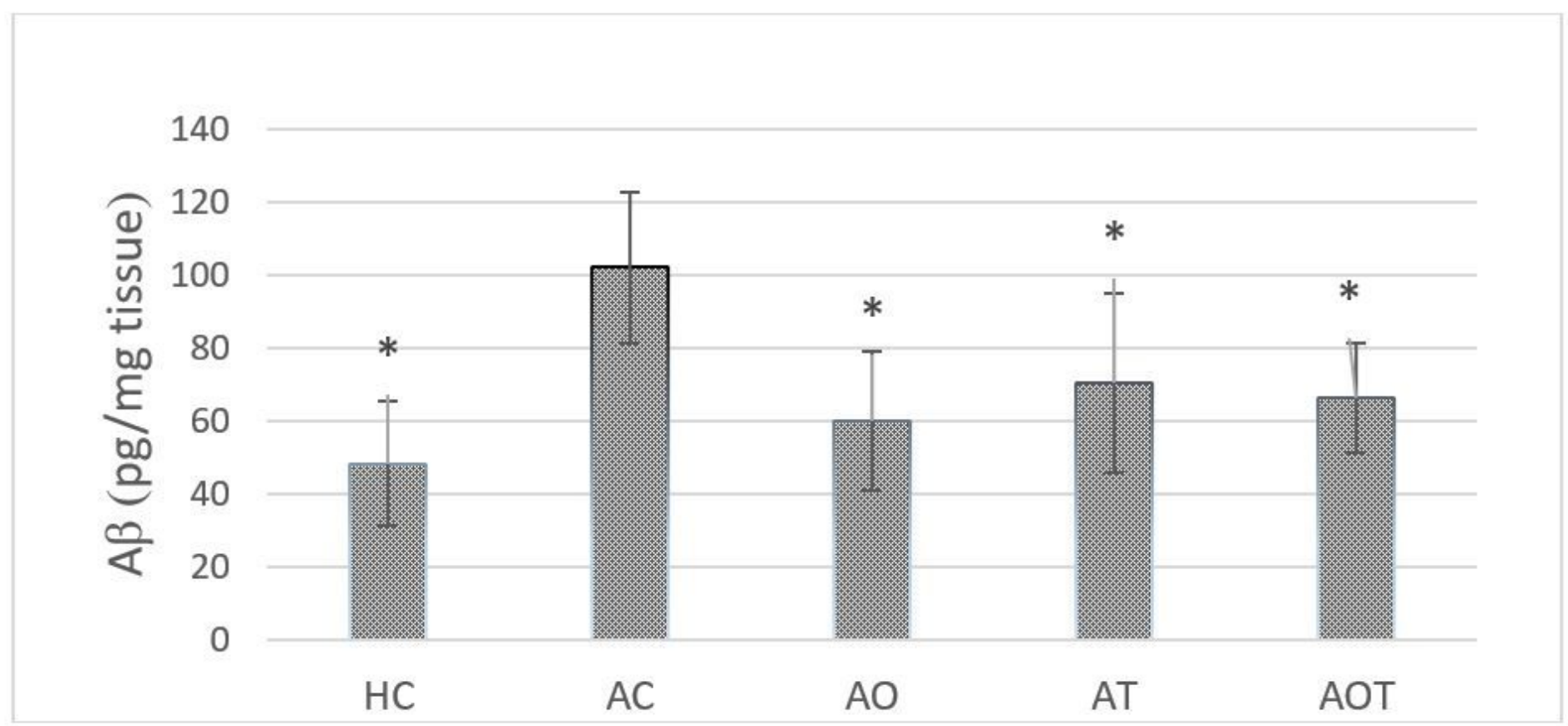

Figure 1

Aß42 levels in the hippocampus of groups. * Significant difference than AC group in P®0.05.

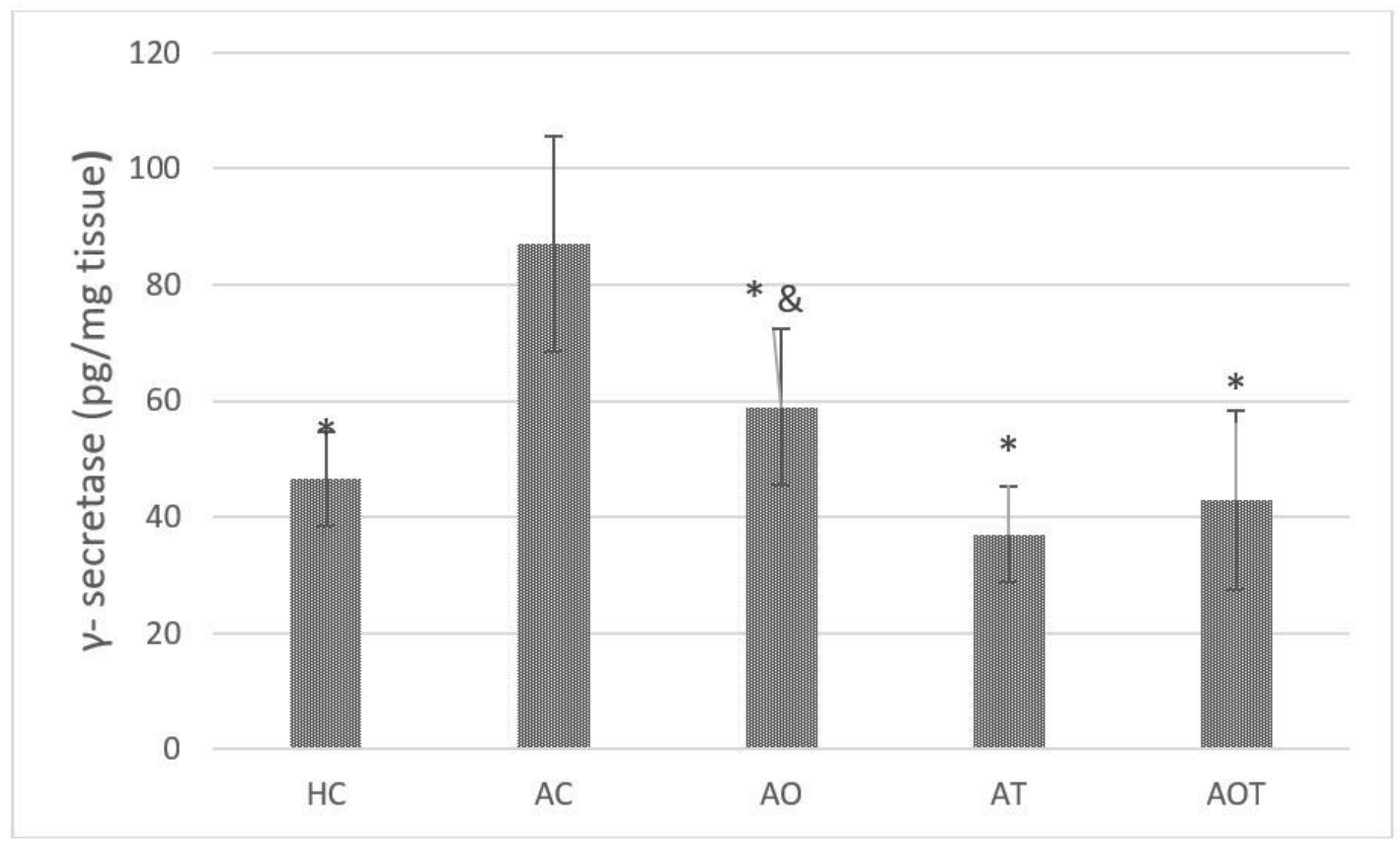


Figure 2

$Y$ - secretase levels in the hippocampus of research groups. * Significant difference than AC group and \& significant difference than AT group in Pख0.05.

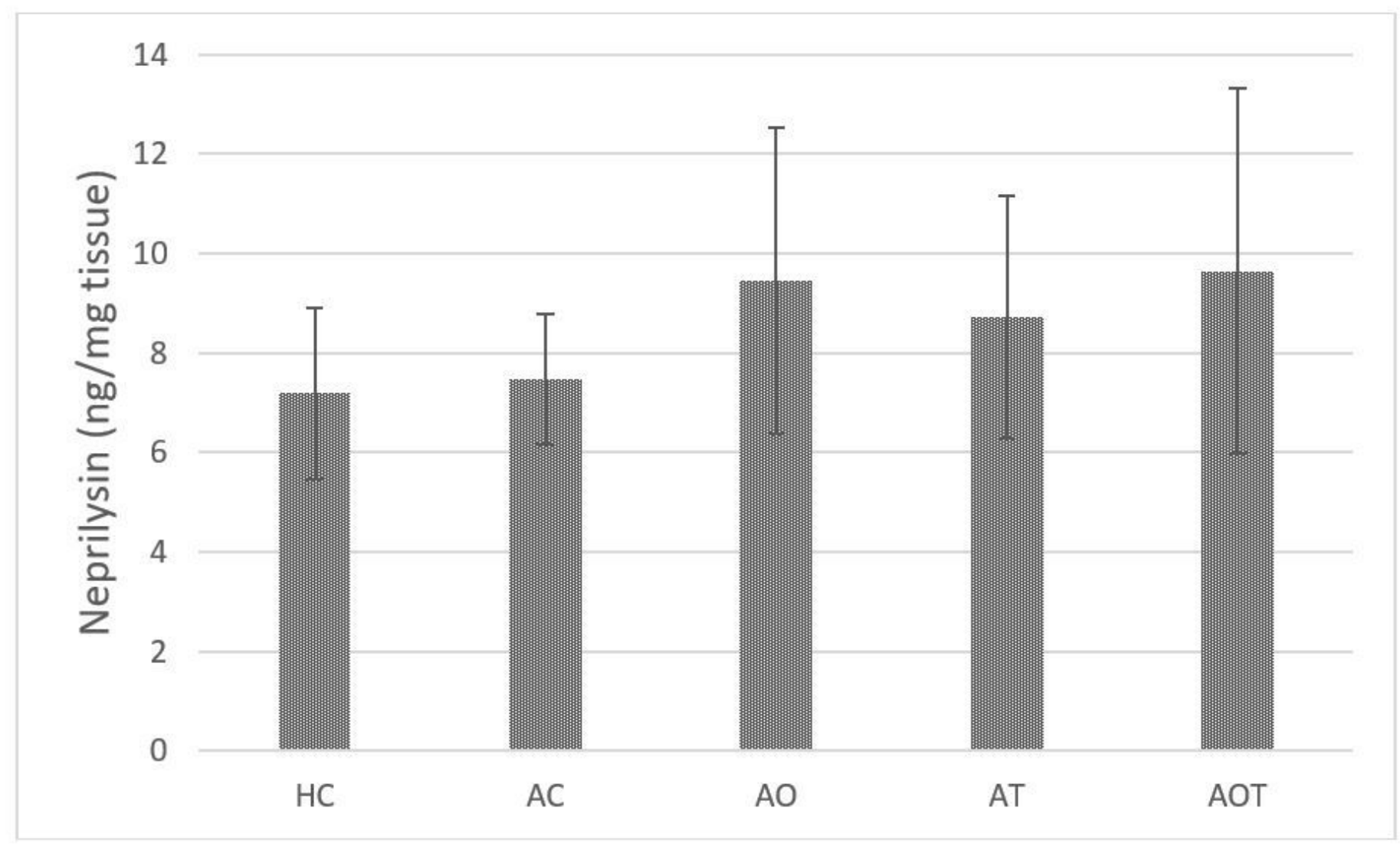

Figure 3

Neprilysin levels in the hippocampus of research groups. 